\title{
A NOTE ON ENGEL GROUPS AND LOCAL NILPOTENCE
}

\author{
R. G. BURNS and YURI MEDVEDEV
}

(Received 6 September 1996; revised 4 July 1997)

Communicated by R. B. Howlett

\begin{abstract}
This paper is concerned with the question of whether $n$-Engel groups are locally nilpotent. Although this seems unlikely in general, it is shown here that it is the case for the groups in a large class $\mathscr{C}$ including all residually soluble and residually finite groups (in fact all groups considered in traditional textbooks on group theory). This follows from the main result that there exist integers $c(n), e(n)$ depending only on $n$, such that every finitely generated $n$-Engel group in the class $\mathscr{C}$ is both finite-of-exponent- $e(n)$-bynilpotent-of-class $\leq c(n)$ and nilpotent-of-class $\leq c(n)$-by-finite-of-exponent- $e(n)$. Crucial in the proof is the fact that a finitely generated Engel group has finitely generated commutator subgroup.
\end{abstract}

1991 Mathematics subject classification (Amer. Math. Soc.): 20F45, 20F19, $20 \mathrm{E} 10$.

\section{Introduction}

A group is called Engel if for each ordered pair $(g, h)$ of elements of the group there is a relation of the form

$$
[\ldots[[g, h], h], \ldots, h]=1 \text {, }
$$

where $[x, y]:=x^{-1} y^{-1} x y$, the commutator of $x$ and $y$. Following the usual 'leftnormed' convention, we write the left-hand side of $(1)$ as $[g, h, \ldots, h]$, or even more briefly as $\left[g,{ }_{n} h\right]$, where $n$ denotes the number of entries of $h$. The Engel condition (1) represents a generalization of local nilpotence: a locally nilpotent group is (clearly) Engel. Essentially the only known examples of non-locally nilpotent Engel groups are those of Golod (see [8, p. 132]): for each $d \geq 2$ there is a $d$-generator nonnilpotent group $G_{d}$ each of whose $(d-1)$-generator subgroups is nilpotent (and $G_{d}$ is in addition residually finite and a $p$-group). It is interesting to contrast with this the known positive results: of Wilson and Zelmanov [12, Theorem 5] that a profinite Engel

(C) 1998 Australian Mathematical Society 0263-6115/98 \$A2.00+0.00 
group is locally nilpotent, of Baer (see [10, p. 360]) that an Engel group satisfying the maximal condition is nilpotent, and of Gruenberg [3] that a soluble Engel group is locally nilpotent.

However it is unknown whether or not $n$-Engel groups, that is, those satisfying the law $\left[x,{ }_{n} y\right] \equiv 1$ for some fixed $n$, must be locally nilpotent (although this seems unlikely). This has been established for $n \leq 3$ (see [6]), and, for general $n$, for the class of residually finite $n$-Engel groups [11]. (Note that there are relatively easy examples of non-nilpotent $n$-Engel groups; see, for example, [8, p. 132] or [10, p. 362, Ex. 1].)

In the present note we call attention to a simple general fact about Engel groups which has apparently hitherto gone unnoticed, and from it infer firstly the local nilpotence of Engel 'SB-groups' (these are defined below; they include soluble groups), and then a quite specific global description of the $n$-Engel groups in a large class $\mathscr{C}$ of groups (including soluble and residually finite groups), yielding in particular their local nilpotence.

The 'simple fact' in question is as follows:

PROPOSITION. A finitely generated Engel group $G$ has finitely generated commutator subgroup $[G, G]$. Moreover if $G$ is $d$-generator and $n$-Engel, then the rank of $[G, G]$ is bounded in terms of $d$ and $n$.

It is immediate that a finitely generated soluble Engel group is polycyclic, and therefore, in view of Baer's result mentioned above, nilpotent. In fact this argument applies to the larger class of 'SB-groups', defined as follows: An SB-group $G$ is a group with a subnormal series

$$
G=G_{0} \unrhd G_{1} \unrhd \cdots \unrhd G_{k}=\{1\},
$$

each of whose factors $G_{i} / G_{i+1}$ is either soluble or locally finite of finite exponent, that is, $\mathrm{G}$ lies in a product $\mathfrak{S}_{1}, \mathfrak{B}_{\ell_{1}} \cdots \mathfrak{S}_{l}, \mathfrak{B}_{e_{r}}$ of varieties, where $\mathfrak{S}_{l}$ denotes the variety of all soluble groups of length $\leq l$ and $\mathfrak{B}_{e}$ the variety consisting of all locally finite groups of exponent dividing $e$. (That the class $\mathfrak{B}_{e}$ is actually a variety is a consequence of Zelmanov's solution of the restricted Burnside problem.) Thus we have the

COROLLARY 1 (Cf. Gruenberg [3]). An Engel SB-group (in particular a soluble Engel group) is locally nilpotent.

The above-mentioned class $\mathscr{C}$, originally introduced in [1], is obtained from the class of all SB-groups by closing under the operations $L$ and $R$, where for any grouptheoretical class $\mathscr{X}, L \mathscr{X}$ denotes the class of all groups locally in $\mathscr{X}$, and $R \mathscr{X}$ the class of all groups residually in $\mathscr{X}$.

Our main theorem is then as follows: 
THEOREM. There exist integers $c(n), e(n)$ depending on $n$ only such that all $n$-Engel groups contained in the class $\mathscr{C}$ are actually contained in the variety

$$
\mathfrak{V}_{n}:=\mathfrak{N}_{c(n)} \mathfrak{B}_{e(n)} \cap \mathfrak{B}_{e(n)} \mathfrak{N}_{c(n)},
$$

where $\mathfrak{N}_{c}$ denotes the variety of all groups nilpotent of class $\leq c$.

Note however that there are Engel (as opposed to $n$-Engel) groups in the class $\mathscr{C}$ which are not locally nilpotent, as is shown by the above-mentioned examples of Golod.

This theorem includes, in particular, the result of Gruenberg [4] that an $n$-Engel soluble group of derived length $d$ must belong to $\mathfrak{B}_{\hat{e}(n)} \mathfrak{N}_{\hat{c}(d, n)}$ for some positive integers $\hat{e}(n)$ and $\hat{c}(d, n)$. Note also that a result of Groves [2, Theorem C] implies a similar conclusion to that of our theorem for $n$-Engel groups lying in a product of a succession of soluble or Cross varieties. The most significant improvement, in our theorem, over these results consists in the dependence of the nilpotency class $c(n)$ and the exponent $e(n)$ exclusively on the Engel class $n$. (Compare the result of Heineken [6] and Gupta and Newman [5] that every 3 -Engel group belongs to $\mathfrak{B}_{20} \mathfrak{N}_{5}$. No such precise facts appear to be known for 4-Engel groups, not even whether they are all locally nilpotent.) Our theorem also generalizes [1, Corollary 2] stating in part that a residually finite, torsion-free, $n$-Engel group is nilpotent of class bounded in terms of $n$ : by the theorem any $n$-Engel group from the class $\mathscr{C}$, and so in particular any residually finite $n$-Engel group, is nilpotent of class bounded in terms of $n$, modulo a normal subgroup of finite exponent.

The local nilpotence of $n$-Engel groups in $\mathscr{C}$ follows easily from the theorem; we leave the details to the reader:

COROLLARY 2. The n-Engel groups in the class $\mathscr{C}$ are locally nilpotent.

The proof of the above theorem is given in Section 2, and of the proposition in Section 3.

REMARK. As noted in [1], it seems reasonable to suggest the class $\mathscr{C}$ as comprising just those groups accessible to analysis using what might be called the 'classical' methods of group theory (such as those used in the textbooks [10] and [8]), in contrast with those outside $\mathscr{C}$ which, one may conjecture, require the quite distinct 'industrial' techniques of, most notably, Adjan-Novikov and Ol'shanskiǐ, used in connection with the negative solution of the general Burnside problem and related problems, and involving the construction of 'monsters'. 


\section{Proof of the Theorem}

Let $G$ be an $n$-Engel group in the class $\mathscr{C}$. We wish to show that $G$ lies in the intersection (2), that is, in the variety $\mathfrak{V}_{n}$.

We firstly prove that $G$ belongs to $\mathfrak{N}_{c_{1}(n)} \mathfrak{B}_{\ell_{1}(n)}$ for some $c_{1}(n), e_{1}(n)$ depending on $n$ only. If we can prove this for an arbitrary finitely generated $n$-Engel SB-group then it will follow for every $n$-Engel group in $\mathscr{C}$ in view of this exclusive dependence on $n$. Hence we may assume without loss of generality that $G$ is a finitely generated SB-group. Then by Corollary 1 above $G$ must be nilpotent, and therefore certainly residually finite. Now it follows from a theorem of Wilson [11, Theorem 2] that every 2 -generator subgroup of a residually finite $n$-Engel group is nilpotent of class bounded in terms of $n$ alone, and hence, according to Mal'cev [9], such a group satisfies a (2variable) semigroup law of degree depending only on $n$. Hence our group $G$ satisfies such a semigroup law, whence by [1, Theorem A], we have

$$
G \in \mathfrak{N}_{c_{1}(n)} \mathfrak{B}_{e_{1}(n)},
$$

for some $c_{1}(n), e_{1}(n)$ depending only on $n$, as required.

We now complete the proof of our theorem by deducing from (3), just established, together with the assumption that $G$ is finitely generated and $n$-Engel, that

$$
G \in \mathfrak{B}_{e_{2}(n)} \mathfrak{N}_{c^{2}(n)},
$$

for some $e_{2}(n), c_{2}(n)$ depending on $n$ only. We proceed by induction on the parameter $c_{1}$ in (3). The initial case $c_{1}=0$ (that is, $G \in \mathfrak{B}_{e_{1}}$ ) is trivial; suppose that $c_{1}>0$ and inductively that a containment of the form (4) holds for classes $<c_{1}$. Set

$$
H:=\gamma_{c_{1}}\left(G^{e_{1}}\right):=[\underbrace{G^{e_{1}}, \ldots, G^{e_{1}}}_{c_{1}}] \unlhd G .
$$

By (3) $H$ is contained in the centre of $G^{e_{1}}$, so that $H$ is certainly abelian. Since $G / H \in \mathfrak{N}_{c_{1}-1} \mathfrak{B}_{e_{1}}$, we may assume by the inductive hypothesis that $G / H \in \mathfrak{B}_{e_{3}} \mathfrak{N}_{c_{3}}$ for some functions $e_{3}=e_{3}(n), c_{3}=c_{3}(n)$ of $n$ only.

The next step in the proof requires the following

LEMMA 1. For each $x \in G, h \in H$, and positive integer $k$, we have

$$
[h, x]^{e_{1}^{k}}=\left[h,{ }_{i} x\right]^{ \pm 1} \cdots\left[h,{ }_{i,} x\right]^{ \pm 1}
$$

for some $t \geq 1, i_{1}, \ldots, i_{t} \geq k$. 
PROOF. It suffices to show that for every $i \geq 1$,

$$
\left[h,{ }_{i} x\right]^{e_{1}}=\left[h, j_{1} x\right]^{ \pm 1} \cdots\left[h, j_{1} x\right]^{ \pm 1}
$$

for some $t \geq 1, j_{1}, \ldots, j_{t} \geq i+1$. The equation (5) follows from this by means of an easy induction on $k$, using the facts that $H$ is normal in $G$ and abelian.

For $i=1,(6)$ has the form

$$
[h, x]^{e_{1}}=\left[h, j_{1} x\right]^{ \pm 1} \cdots\left[h, j_{i} x\right]^{ \pm 1}, j_{1}, \ldots, j_{t} \geq 2 .
$$

This follows by repeated application of the group identity $[a, b c] \equiv[a, c][a, b][a, b, c]$, invoking the abelian-ness and normality in $G$ of $H$ : thus

$$
\begin{aligned}
& {\left[h, x^{2}\right]=[h, x]^{2}[h, x, x],} \\
& {\left[h, x^{3}\right]=\left[h, x^{2}\right][h, x]\left[h, x, x^{2}\right]=[h, x]^{3}[h, x, x]^{3}[h, x, x, x],}
\end{aligned}
$$

and so on, whence, eventually,

$$
\left[h, x^{e_{1}}\right]=[h, x]^{e_{1}}\left[h, j_{1} x\right] \cdots\left[h, j_{1} x\right]
$$

where $j_{1}, \ldots, j_{t} \geq 2$. Since $x^{e_{1}}$ commutes with $h$, an equation of the form (7) follows.

Now suppose that $i \geq 1$ and inductively that (6) holds with $i-1$ in place of $i$. We have

$$
\left[h,{ }_{i} x\right]^{e_{1}}=\left[h, x,{ }_{i-1} x\right]^{e_{1}}=\left[h_{1}, i_{i-1} x\right]^{e_{1}},
$$

where $h_{1}:=[h, x] \in H$. The inductive hypothesis then gives

$$
\left[h_{1}, i_{i-1} x\right]^{e_{1}}=\left[h_{1},{ }_{1} x\right]^{ \pm 1} \cdots\left[h_{1}, l_{1} x\right]^{ \pm 1},
$$

where $r \geq 1, l_{1}, \ldots, l_{r} \geq i$. Since $h_{1}=[h, x]$, the desired conclusion $(6)$ follows for $i$.

Returning to the proof of the theorem, we conclude from this lemma and the assumption that $G$ is $n$-Engel, that $[G, H]^{e_{1}^{n}}=\{1\}$. Since this exponent depends only on $n$, we may work modulo $[G, H]$, that is, we may assume without loss of generality that $H$ is central in $G$. Thus to summarize, we are now in the situation of a finitely generated $n$-Engel group $G$ with a central subgroup $H$ such that

$$
G / H \in \mathfrak{N}_{c_{1}-1} \mathfrak{B}_{e_{1}} \cap \mathfrak{B}_{e_{3}} \mathfrak{N}_{c_{3}},
$$

and we seek to establish (4) for such a group G. As noted before, we also have by [11, Theorem 2] that every 2-generator subgroup of $G$ is nilpotent of class $\leq c_{4}$ for some $c_{4}$ depending only on $\mathrm{n}$.

To conclude the proof we shall need the following 
LEMMA 2. Let $G$ be as above. Then for any $x \in \gamma_{c_{3}}(G), g \in G$, the commutator subgroup $[\langle x, g\rangle,\langle x, g\rangle]$ of $\langle x, g\rangle$, has exponent dividing $e_{3}^{c_{4}-1}$.

PROOF. Since by [11, Theorem 2] the 2-generator subgroup $\langle x, g\rangle$ is nilpotent of class $\leq c_{4}$, it suffices to show that for each $i \geq 2$ the quotient $\gamma_{i}(\langle x, g\rangle) / \gamma_{i+1}(\langle x, g\rangle)$ has exponent dividing $e_{3}$. Now by definition $\gamma_{i}(\langle x, g\rangle)$ is generated by the commutators of the form $\left[x_{1}, g_{1}\right], x_{1} \in \gamma_{i-1}(\langle x, g\rangle), g_{1} \in\langle x, g\rangle$. One has

$$
\left[x_{1}, g_{1}\right]^{e_{3}} \equiv\left[x_{1}^{e_{3}}, g_{1}\right] \bmod \gamma_{i+1}(\langle x, g\rangle)
$$

and then since $x_{1} \in \gamma_{c_{3}}(G)$, which has exponent dividing $e_{3}$ modulo the centre of $G$, we have that $\left[x_{1}^{e_{3}}, g_{1}\right]=1$, whence the lemma.

Using this lemma we shall now show that

$$
\gamma_{c_{3}+1}(G)^{c_{4} ! e_{3}^{c_{4}}}=1
$$

From this the desired conclusion (4) follows, with $c_{2}=c_{3}+1$, and $e_{2}$ some more complicated function of $e_{1}, n, e_{3}, c_{4}$, and so ultimately of $n$ alone.

Write $e_{4}:=c_{4} ! e_{3}^{c_{4}}$. By definition of $\gamma_{c_{3}+1}(G)$ each element of that group is a product $a_{1} \cdots a_{t}$ of commutators $a_{i}$ of the form $[x, g]^{ \pm 1}, x \in \gamma_{c_{3}}(G), g \in G$. We prove by induction on $t$ that every such product has order dividing $e_{4}$, that is,

$$
\left(a_{1} \cdots a_{t}\right)^{e_{4}}=1 .
$$

For $t=1$ this follows from Lemma 2, since by that lemma any element of the form $[x, g], x \in \gamma_{c_{3}}(G), g \in G$, has order dividing $e_{3}^{c_{4}}$, which in turn divides $e_{4}$. Suppose that $t>1$ and inductively that the analogue of (9) holds for such products of length $<t$. Write $a:=a_{1}, b:=a_{2} \cdots a_{t}$. By the Hall-Petrescu identity (see, for example, [7, p. 317, Satz 9.4])

$$
a^{e_{4}} b^{e_{4}}=(a b)^{e_{4}} w_{2}(a, b) \cdots w_{e_{4}}(a, b)
$$

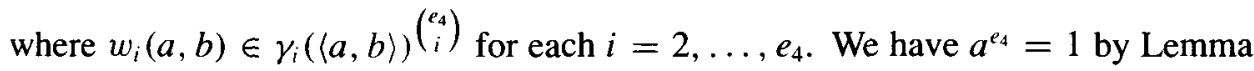
2 , and $b^{e_{4}}=1$ by the inductive hypothesis. If $i>c_{4}$ then $\gamma_{i}(\langle a, b\rangle)=\{1\}$ since, as noted earlier, every 2-generator subgroup of $\mathrm{G}$ has class $\leq c_{4}$. On the other hand if $2 \leq i \leq c_{4}$, then it is easy to see that $\left(\begin{array}{c}e_{4} \\ i\end{array}\right)$ is divisible by $e_{3}^{c_{4}}$, so that for these $i$ we have $\gamma_{i}(\langle a, b\rangle){ }^{\left({ }^{e_{4}}\right)}=\{1\}$, by Lemma 2. Thus for all $i=2, \ldots, e_{4}$, we have $w_{i}(a, b)=1$. Hence $(a b)^{e_{4}}=1$, completing the induction, and thence the proof of the theorem. 


\section{Proof of the Proposition}

We firstly show that if $G$ is any Engel group, then for any $x, y \in G$, the subgroup $\langle x\rangle^{(y)}$ is finitely generated. (This appears as Exercise 6 on p. 362 of [10]; we include a proof for completeness, since the proposition is crucial in the above argument.)

Write $x_{i}:=y^{i} x y^{-i}$ for each integer $i$. We show by induction on $n$ that $\left[x,{ }_{n} y\right]$ has the form

$$
\left[x,{ }_{n} y\right]=u_{n} x_{0}^{ \pm 1} v_{n} x_{-n}^{ \pm 1},
$$

for some words $u_{n}, v_{n}$ in $x_{-1}, \ldots, x_{-(n-1)}$. For $n=1$ we have $[x, y]=x^{-1} y^{-1} x y=$ $x_{0}^{-1} x_{-1}$, which has the right form with $u_{1}=v_{1}=1$. Assuming inductively that (10) holds, we have

$$
\begin{aligned}
{\left[x,{ }_{n+1} y\right] } & =\left[\left[x,{ }_{n} y\right], y\right]=\left(u_{n} x_{0}^{ \pm 1} v_{n} x_{-n}^{ \pm 1}\right)^{-1} y^{-1} u_{n} x_{0}^{ \pm 1} v_{n} x_{-n}^{ \pm 1} y \\
& =\left(x_{-n}^{\mp 1} v_{n}^{-1}\right) x_{0}^{\mp 1}\left(u_{n}^{-1} u_{n}^{y} x_{-1}^{ \pm 1} v_{n}^{y}\right) x_{-(n+1)}^{ \pm 1} .
\end{aligned}
$$

Since $u_{n}^{v}\left(:=y^{-1} u_{n} y\right)$, and $v_{n}^{v}$ are expressions in $x_{-2}, \ldots, x_{-n}$ only, we see that $\left[x,{ }_{n+1} y\right]$ has the appropriate form, completing the induction.

From (10) it follows that if $\left[x,{ }_{n} y\right]=1$, then

$$
x_{-n} \in\left\langle x_{0}, x_{-1}, \ldots, x_{-(n-1)}\right\rangle,
$$

and

$$
x_{0} \in\left\langle x_{-1}, x_{-2}, \ldots, x_{-n}\right\rangle .
$$

Successive conjugations of (11) by y yields

$$
x_{-i} \in\left\langle x_{0}, x_{-1}, \ldots, x_{-n}\right\rangle \text { for all } i>n,
$$

and successive conjugations of (12) by $y^{-1}$ yields

$$
x_{i} \in\left\langle x_{-1}, \ldots, x_{-n}\right\rangle \text { for all } i>0 .
$$

Hence

$$
\langle x\rangle^{(y)}=\left\langle x_{-1}, \ldots, x_{-n}\right\rangle,
$$

showing that $\langle x\rangle^{(y)}$ is indeed finitely generated. It follows that if $H$ is any finitely generated subgroup of $G$ and $g \in G$, then

$$
H^{(g)} \text { is finitely generated. }
$$


The remainder of the proof, that is, the argument deducing from this that any finitely generated subgroup of $G$ has finitely generated commutator subgroup, is identical with that of the proposition of [1]; we reproduce the proof here since, as already noted, the present proposition is crucial to the theorem.

One first shows that given any two elements $a, b \in G$, the commutator subgroup $\langle a, b\rangle^{\prime}$ is finitely generated. The crucial fact allowing this is that $\langle a, b\rangle^{\prime}$ is generated by the elements of the form $[a, b]^{m^{n} b^{n}}$ where $m$ and $n$ are integers. This follows in turn from the well-known fact that $\langle a, b\rangle^{\prime}$ is generated by all commutators of the form $\left[a^{r}, b^{s}\right], r$ and $s$ integers, via repeated application of the identities

$$
\begin{aligned}
& a^{-i}\left[a^{r}, b^{s}\right] a^{i}=\left[a^{r+i}, b^{s}\right]\left[b^{s}, a^{i}\right], \\
& b^{-i}\left[a^{r}, b^{s}\right] b^{i}=\left[b^{i}, a^{r}\right]\left[a^{r}, b^{s+i}\right],
\end{aligned}
$$

starting with $r=s=1$. Now $\langle[a, b]\rangle^{\langle a\rangle}$ is finitely generated by the first part of the proof, whence by $(13)\left(\langle[a, b]\rangle^{\langle a\rangle}\right)^{(b)}$ is finitely generated, as required.

This establishes the 2-generator case. Now assume inductively that the claim is valid for subgroups of $G$ which can be generated by $\leq k$ elements, and suppose that $H<G$ requires $k+1>2$ generators, say $h_{1}, \ldots, h_{n+1}$. Write $H_{i}$ for the subgroup generated by

$$
\left\{h_{1}, \ldots, h_{k+1}\right\} \backslash\left\{h_{i}\right\}, \quad i=1, \ldots, k+1 .
$$

Then by the inductive hypothesis $\left[H_{i}, H_{i}\right]$ is finitely generated, whence so is $\left[H_{i}, H_{i}\right]^{\left(h_{i}\right)}$. The conclusion now follows from the fact that $[H, H]$ is generated by the settheoretical union of the $\left[H_{i}, H_{i}\right]^{\left\langle h_{i}\right\rangle}$. For this it suffices to show that the subgroup generated by this union, that is, by $U:=\bigcup_{i}\left[H_{i}, H_{i}\right]^{\left\langle h_{i}\right\rangle}$, is normal in $H$. For instance

$$
\left(\left[H_{1}, H_{1}\right]^{h_{1}}\right)^{h_{2}}=\left[H_{1}, H_{1}\right]^{h_{2} h_{1}\left[h_{1}, h_{2}\right]}=\left[H_{1}, H_{1}\right]^{h_{1}\left[h_{1}, h_{2}\right]},
$$

and since $\left[H_{1}, H_{1}\right]^{h_{1}} \subseteq U$ and $\left[h_{1}, h_{2}\right] \in\left[H_{3}, H_{3}\right]$, we have $\left[H_{1}, H_{1}\right]^{h_{1} h_{2}} \leq\langle U\rangle$.

\section{References}

[1] R. G. Burns, Olga Macedonska and Yuri Medvedev, 'Groups satisfying semigroup laws, and nilpotent-by-Burnside varieties', J. Algebra 195 (1997), 510-525.

[2] J. R. J. Groves, 'Varieties of soluble groups and a dichotomy of P. Hall', Bull. Austral. Math. Soc. 5 (1971), 391-410.

[3] K. W. Gruenberg, 'Two theorems on Engel groups', Proc. Camb. Phil. Soc. 49 (1953), 377-380.

[4] _ _ 'The upper central series in soluble groups', Illinois J. Math. 5 (1961), 436-466.

[5] N. D. Gupta and M. F. Newman, 'Third Engel groups', Bull. Austral. Math. Soc. 40 (1989), 215-230.

[6] H. Heineken, 'Engelsche Elemente der Länge drei', Illinois J. Math. 5 (1961), 681-707. 
[7] B. Huppert, Endliche Gruppen I (Springer, Berlin, 1967).

[8] M. I. Kargapolov and Ju. I. Merzljakov, Fundamentals of the theory of groups (Springer, Berlin, 1979).

[9] A. I. Mal'cev, 'Nilpotent semigroups', Uchen. Zap. Ivanovsk. Ped. Inst. 4 (1953), 107-111.

[10] D. J. S. Robinson, A course in the theory of groups (Springer, Berlin, 1982).

[11] J. S. Wilson, 'Two-generator conditions in residually finite groups', Bull. London Math. Soc. 23 (1991), 239-248.

[12] J. S. Wilson and E. I. Zelmanov, 'Identities for Lie algebras of pro-p groups', J. Pure Appl. Algebra 81 (1992), 103-109.

Department of Mathematics and Statistics

York University

North York

Ontario, M3J 1P3

Canada

e-mail: rburns@mathstat.yorku.ca

medvedev@mathstat.yorku.ca 